Check for updates

Cite this: RSC Adv., 2017, 7, 27747

\title{
Larger or more? Nanoparticle characterisation methods for recognition of dimers $\uparrow$
}

\author{
D. Mehn, (D) ${ }^{* a}$ F. Caputo, ${ }^{\text {cd }}$ M. Rösslein, ${ }^{\text {b } L . ~ C a l z o l a i, ~}{ }^{a}$ F. Saint-Antonin, ${ }^{c e}$ T. Courant, ${ }^{\text {cd }}$ \\ P. Wick ${ }^{b}$ and D. Gilliland ${ }^{a}$
}

Our article dissects the problem of understanding the origin of size heterogeneity in polydispersed nanoparticle samples. A commercially available multimodal material representing a typical borderline case of the nano definition is characterised with various state of the art techniques. We focus on dimer (multimer) recognition capability of different techniques, considering the potential of single and combined analytical solutions. The performance of dynamic light scattering (DLS), nanoparticle tracking analysis (NTA), online coupled asymmetric field flow fractionation - multi angle light scattering (MALS) DLS (FFF-MALS-DLS), tunable resistive pulse sensing (TRPS), centrifugal liquid sedimentation (CLS), analytical ultracentrifugation (AUC) and transmission electron microscopy (TEM) is discussed. NTA, TRPS and FFF-MALS are shown to resolve the multimodal size distribution of the sample, while batch mode DLS, the most widespread tool in characterisation laboratories, fails. Besides of complex methods like TEM imaging after FFF separation and FFF-MALS-DLS in combination with adequate mathematical shape factor models, centrifugal methods are documented as simple analytical tools that are able to indicate the presence of dimers made of rigid spherical nanoparticles.

Received 27th February 2017

Accepted 17th May 2017

DOI: $10.1039 / \mathrm{c} 7 \mathrm{ra0} 2432 \mathrm{k}$

rsc.li/rsc-advances size distributions, recognising the presence of aggregates or agglomerates can be also necessary to understand whether a certain material should be considered as nanomaterial.

Among the existing techniques measuring nanoparticle size in liquids, dynamic light scattering (DLS) is probably the most widely used. Even if batch mode DLS is well known to have disadvantages for the proper characterisation of polydispersed samples ${ }^{6-8}$ the method becomes very useful when coupled online to prior size separation steps like asymmetric field flow fractionation (FFF). ${ }^{8}$ Calculation of particle size based only on FFF elution times is theoretically possible $e^{9,10}$ but chemical nature of the sample might also have a strong effect on retention times. However, FFF further connected with multi angle light scattering (MALS) and DLS can provide information even on particle morphology by analysing the gyration/hydrodynamic radius ratio of the nanoparticles. ${ }^{\mathbf{1 1}}$

Nanoparticle tracking analysis (NTA or PTA) and Tunable Resistive Pulse Sensing (TRPS) are indirect size-measuring techniques (compared to the direct size measurement by electron microscopy) but still provide directly number based size distributions.

NTA (PTA) is based on the optical observation of light scattering by single objects: particles or particle aggregates in suspension. The Brownian motion of the particles is evaluated, calculating the mean square displacement of each particle in $2 \mathrm{D}$, in order to obtain their translational coefficient and thus, the particle hydrodynamic diameter. The particle by particle tracking method allows resolving individual populations of spherical nanoparticles (NPs) of different sizes by reducing the mehn@ec.europa.eu

${ }^{b}$ Empa, Swiss Federal Laboratories for Materials Science and Technology, St. Gallen, Switzerland

${ }^{c}$ Univ. Grenoble Alpes, F38000 Grenoble, France

${ }^{d}$ CEA, LETI, Minatec Campus, F38054, Grenoble, France

${ }^{e}$ CEA, LITEN, Minatec Campus, F38054, Grenoble, France

$\dagger$ Electronic supplementary information (ESI) available. See DOI: $10.1039 / \mathrm{c} 7 \mathrm{ra02432 \textrm {k }}$ 
effects of scattering light intensities from larger particles, which limits batch scattering methods such as batch mode DLS. ${ }^{12}$ However, this technique does not provide information on particles shape, since the mathematical models assume spherical particles.

Tunable Resistive Pulse Sensing (TRPS) is based on the Coulter principle and uses tunable polyurethane membranes with a tunable nanopore. ${ }^{13-16}$ Nanoparticles are directed through the nanopore by applying both pressure and voltage. Each time a particle is forced to pass through the pore, a resistive pulse signal is detected due to the reduction in ionic current. Since nanoparticles are characterised individually, TRPS gives a number based statistical distribution. TRPS also allows obtaining indirect information on particle shape, by detecting changes in particle speed passing through the pore, which is lower for elongated objects than for spherical particles possessing similar volumes and surface charge. ${ }^{17}$ Centrifugal methods determine the size of sedimenting particles based on their sedimentation speed. Centrifugal liquid sedimentation (CLS) detects light extinction and calculates equivalent sphere diameters from sedimentation times applying the Stokes equation. As a result it provides extinctionbased size distributions that can be further transformed to mass or number based distributions applying the Mie theory and using absorption, refractive index, and particle density as input parameters. $^{18,19}$ Instead, analytical ultracentrifugation (AUC) is able to generate sedimentation coefficient distributions from interference measurements. ${ }^{20}$ This can be transformed to mass and number based distributions needing only density as input parameter. Both centrifugation techniques determine Stokes diameter of an equivalent sphere (diameter of a sphere that sediments with the same speed) but have the potential to use more complex models including a shape factor (or frictional coefficient ratio in case of AUC) that considers the effect of particle geometry on the sedimentation speed. ${ }^{21}$

Transmission electron microscopic (TEM) is a highly valuable technique for the size analysis of nanomaterials as it is possible to obtain a direct image of the particles. Image analysis softwares facilitate the statistical analysis of the data, providing information on particle shape and number based size distributions. However, the particles should be well dispersed and randomly distributed on the grid. Unfortunately, simple TEM sample preparation methods (by evaporation of the solution) do not distinguish between agglomerates formed during the drying of the sample aliquot on the support (grid) and aggregates already present in the NP suspension. Cryo-TEM might help to overcome this drawback by an ultra-fast conversion of the NP suspension into a vitrified film on the grid, allowing direct morphological visualization of nanomaterials at near native state, and thus, reducing the risk of aggregation and of the introduction of artefacts due to sample preparation. However, interactions with the carbon support of the grid may induce a preferential orientation of some specific samples on the grid. Moreover, the technique needs laborious sample preparation, and the maximum size of an object to be observed is limited by the thickness of the vitrified film, which is about $300 \mathrm{~nm}$, and thus large particles or aggregates cannot be imaged as the technique lead to their removal if any were present in the sample. ${ }^{22}$
Strategies to gain reliable number based particle size distribution (PSD) of multimodal mixtures of particles of different sizes were investigated by many, mostly by intentionally mixing standard polystyrene beads ${ }^{7}$ or metallic particles ${ }^{23}$ in known proportions. Some recent works started to focus on real life samples, such as polymeric NPs, ${ }^{6}$ mixture of exosomes ${ }^{24}$ or marketed consumer products such as colloidal silver. It was shown that detecting the PSD of such complex dispersions requires a combination of suitable number based methods (TEM, NTA (PTA), TRPS), or introduction of a separation step (e.g. FFF, centrifugation) prior to size detection by light scattering. ${ }^{6,25}$

On the other hand, ways to detect dimers and multimers made of rigid spherical particles of the same size were much less investigated. Simonsen et $a .^{23}$ very recently proposed the use of flow cytometry as a separation step, combined with light scattering to determine the distribution of single particle, dimers and multimers of gold NPs. However, the proposed approach is limited to highly scattering metallic particles of size $>50-70 \mathrm{~nm}$, and it is not compatible with NPs possessing refractive index similar to water, such as liposomes or polymeric micelles, where the lower detectable size would be much larger than $100 \mathrm{~nm} .{ }^{23}$ Thus, correctly assessing the presence of small aggregates, such as dimers and multimer nanostructures in complex mixtures is still an open challenge to be addressed. In our work, we describe the performance of the above mentioned analytical techniques that can help to uncover aggregates and are actually available as typical state of the art instrumentation in material science laboratories.

\section{Experimental}

Aminated polystyrene nanoparticles (PA02N) with bimodal size distribution and a mean diameter of $0.12 \mu \mathrm{m}$ were purchased from Bangs laboratories Inc, (9025 Technology Dr Fishers, IN, USA). The sample contained surfactant (0.2\% SDS) as stabiliser. All other chemicals were purchased from Sigma Aldrich. Buffers were filtered through a $0.22 \mu \mathrm{m} 500 \mathrm{~mL}$ Corning ${ }^{\circledR}$ PES sterile Filter System before use.

Batch mode DLS measurements were performed using a Malvern Zetasizer Nano-ZS instrument equipped with a $633 \mathrm{~nm} \mathrm{HeNe}$ laser. The original particle suspension was diluted 100 times in the solvent (water or $0.08 \% \mathrm{NaOH}+0.02 \%$ SDS in water) and equilibrated for 5 minutes before the measurements at $25{ }^{\circ} \mathrm{C}$. Size distribution results were generated by averaging 10 consecutive measurements of 12 times $10 \mathrm{~s}$ runs. A refractive index value of 1.485 and absorption of 0.01 were considered during the calculation of number based size distributions.

Nanoparticle tracking analysis was done using a Malvern Nanosight instrument NS500-Z equipped with a $532 \mathrm{~nm}$ green laser and sCMOS camera. The original particle suspension was diluted 50000 times in MilliQ water, and injected to the analysis chamber using a syringe pump at $26{ }^{\circ} \mathrm{C}$. The particle size distribution was calculated by averaging 3 times 10 consecutive measurements, each of them based on average 10500 completed tracks.

TRPS measurements were performed on an Izon QNano Gold (Izon Science Ltd., Christchurch, New Zealand) with 
a CPC100 nanopore and a measurement window of 40-230 nm. Phosphate buffered saline containing $0.03 \%(\mathrm{w} / \mathrm{w})$ of Tween ${ }^{\circledR} 20$ was used as electrolyte and dispersion medium for all dilutions and experiments. The nanopore was first stretched to $47.00 \mathrm{~mm}$ and wetted with electrolyte for one minute under a pressure of $2000 \mathrm{~Pa}$. Stretch was then optimized at $45.05 \mathrm{~mm}$ with an applied voltage of $0.96 \mathrm{~V}$ for calibration particles. Calibration was made using carboxylated polystyrene nanoparticles (CPC100, Izon Sciences) at a dilution of 1/1000 (v/v) under a pressure of $400 \mathrm{~Pa}$. (According to the manufacturer, the calibration particles had a mean diameter of $110 \mathrm{~nm}$, a mode diameter of $110 \mathrm{~nm}$ and a concentration of $1.1 \times 10^{13}$ particles per $\mathrm{mL}$.) For the samples recording, PA02N was diluted to $1 /$ $1000(\mathrm{v} / \mathrm{v})$ and measured in triplicate under a pressure of 400 $\mathrm{Pa}$, which was kept constant during the measurements. A minimum of 1000 events were recorded for each replicate to obtain a good representation of particle size distribution. At the end of the measurements, a final calibration measurement was performed and compared to the first calibration in order to check that no significant change occurred on the nanopore during the global experiment. Particle size distribution was calculated using Izon Control Suite Software v 3.2.2.268. The raw data were exported and plotted using Origin 8.0 software.

A Postnova AF4 asymmetric field flow fractionation system equipped with an absorbance detector and online connected to a Malvern Zetasizer Nano-ZS instrument and a Wyatt Dawn Helios multi angle light scattering (MALS) detector were used to realise FFF-UV-Vis-MALS-DLS measurements. The particle suspension diluted ten times in MilliQ water was injected through a $20 \mu \mathrm{L}$ loop into the FFF channel (350 $\mu \mathrm{m}$ spacer, 10 $\mathrm{kDa}$ regenerated cellulose membrane). $\mathrm{NaOH}$ solution $(0.08 \%)$ containing $0.02 \%$ SDS was applied as mobile phase at $0.5 \mathrm{~mL}$ $\min ^{-1}$ flow, $1.25 \mathrm{~mL} \min ^{-1}$ crossflow and exponentially decreasing crossflow profile. Absorbance of the eluted fractions was monitored at $230 \mathrm{~nm}$. Hydrodynamic and geometric diameters of the particles were determined at the maximum of the absorption based elugram from the on-line DLS and MALS measurements, respectively. FFF fractions were collected using a Postnova automatic fraction collector.

CLS measurement was performed in a CPS disc centrifuge equipped with a $405 \mathrm{~nm}$ laser, at $22000 \mathrm{rpm}$, using a $0-8 \%(\mathrm{w} / \mathrm{w})$ sucrose gradient containing $0.2 \%(\mathrm{w} / \mathrm{v})$ SDS. An aliquot of $100 \mu \mathrm{L}$ 100 times diluted particle suspension was injected in the disk of the centrifuge after calibration with $239 \mathrm{~nm}$ PVC particles. A refractive index value of 1.624 , absorption of 0.001 and density of $1.05 \mathrm{~g} \mathrm{~mL}^{-1}$ were used as input parameters for the calculations.

AUC analysis of the ten times dilutes sample (in MilliQ water) was performed using a Beckman Coulter ProteomeLabTM XL-I analytical ultracentrifuge with interference optics. The sample holder was placed in an 8 holes rotor and measurements were run at a constant rotation speed of $5000 \mathrm{rpm}$ at $20{ }^{\circ} \mathrm{C}$. The ls$\mathrm{g}^{*}(\mathrm{~s})$ model of the Sedfit software ${ }^{26}$ was applied to fit experimental data to calculate sedimentation coefficient distribution. The sedimentation coefficient distribution was transformed to mass based size distribution using the "transform s distribution to $r$ distribution" option of Sedfit.
Cryo-TEM images were taken using a Tecnai Osiris (FEI) operated at $200 \mathrm{kV}$. The NPs were first diluted 10 times in SDS $0.05 \%$. Then, $2 \mu \mathrm{L}$ drop of the diluted suspension was delivered on a Agar C-166-3 lacey carbon grid. The sample was automatically Cryo-vitrified using a Vitrobot (FEI) and with the help of liquid ethane (at about $-180^{\circ} \mathrm{C}$ ). The images were processed by scoring the size of 300 not overlapping NPs. Particle size distribution was obtained plotting the Feret diameter obtained for each particle, making use of Image J software. TEM analysis of the separated AF4 fractions was performed after centrifugation of the $1 \mathrm{~mL}$ aliquots in Eppendorf tubes for $20 \mathrm{~min}$ at $10000 \mathrm{rpm}$ followed by resuspension in MilliQ water. Alcyanblue treated ultrathin Formvar-coated 200-mesh copper grids were applied in order to avoid aggregate formation during the sample preparation. The grid was placed on top of the sample drop and left there for 10 minute to allow adsorption of particles on the grid. Then the excess of liquid was removed and the grids were left to dry at $4{ }^{\circ} \mathrm{C}$. Images were taken using a JEOL 2100 transmission electron microscope at an accelerating voltage of $200 \mathrm{kV}$.

Long term stability of the particle suspension was verified by FFF separation and CPS measurements 17 months after the first experiments showing no change in particle size distribution (results not shown).

\section{Results and discussion}

\section{Nanoparticle tracking analysis}

The optical tracking of the particle suspension clearly revealed a bimodal size distribution of the analysed material with prominent modes at 95 and $133 \mathrm{~nm}$ and a shoulder indicating the presence of larger particles above $250 \mathrm{~nm}$ (Fig. 1A).

According to the Nanosight, the sample has a number based size distribution median of $135 \pm 0.7 \mathrm{~nm}$ - classifying it as "non-nano" material (according to the EC recommended definition).

\section{Batch mode DLS}

Batch mode DLS can be successfully applied in some cases to resolve multimodal particle size distributions. ${ }^{27}$ However, the performance of DLS strongly depends on the size difference between the modes. As observed earlier in case of multimodal samples in similar size range, ${ }^{6,7}$ batch mode DLS was not able to recognise the bimodal nature of the sample, providing a single peak at $120.5 \mathrm{~nm}$ (mean) in the intensity based distribution and $84.5 \mathrm{~nm}$ (mean) in the number based distribution (Fig. 1B), a $Z$ average of $111.4 \mathrm{~nm}$ and a quite low polydispersity index of 0.07 . Nevertheless, batch mode DLS was applied as fast and simple method to check the integrity of particles in the mobile phase of the FFF-UV-Vis-MALS-DLS system. The overlay of the DLS analysis of the samples diluted in MilliQ water and $0.08 \% \mathrm{NaOH}$ (Fig. 1B) shows perfect match, suggesting that no degradation or particle aggregation is expected to happen in the selected mobile phase. 


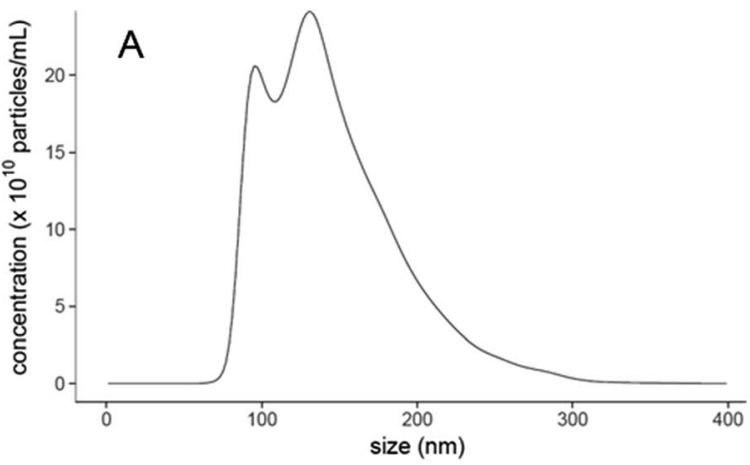

B

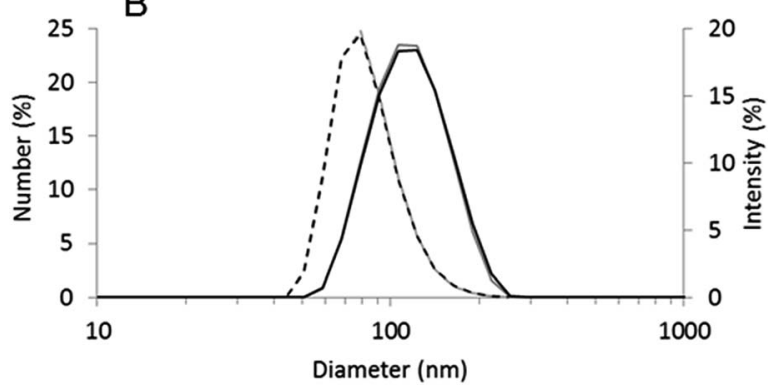

Fig. 1 (A) NTA (PTA) analysis results of the sample diluted in water. (B) DLS results of the sample diluted in water (black lines) and the particles in $0.08 \% \mathrm{NaOH}+0.02 \%$ SDS (grey lines) solid lines: intensity based, dashed lines: number based distributions.

\section{FFF-UV-Vis-MALS-DLS}

Mobile phase with a $\mathrm{pH} 12.5$ and containing $0.02 \%$ SDS was applied in the FFF separation method in order to avoid the adsorption of the particles on the negatively charged regenerated cellulose membrane. Zeta potential of the particle suspension diluted in $10 \mathrm{mM}$ phosphate buffer was about $-40 \mathrm{mV}$ that was shifted to $-60 \mathrm{mV}$ in the $0.08 \% \mathrm{NaOH}$ solution (ESI, Fig. S2 $\dagger$ ). Optimisation of the eluent buffer, focusing time and cross-flow profile (ESI, Fig. S3†) allowed the separation of the two main components of the particle suspension (Fig. 2) with acceptably short elution time, and well resolved peaks in the elugram. In contrast to the NTA results, UV-Vis intensity of the two particle populations suggests that the smaller particles are present in higher number in the sample. Geometric radii $\left(R_{\mathrm{G}}\right)$ calculated based on the MALS data by fitting with the sphere model resulted in $42.5 \pm 0.3 \mathrm{~nm}$ and $66.9 \pm 0.3 \mathrm{~nm}$ at the two absorption peak maxima.

Online DLS measurements in the same experiment (Fig. 2) indicated hydrodynamic diameter $\left(D_{\mathrm{H}}\right)$ values of 92 and $123 \mathrm{~nm}$ for the two particle populations. The calculated shape factors $\left(2 R_{\mathrm{G}} / D_{\mathrm{H}}\right)$ for the first and second component are 0.92 and 1.08, respectively. While the ratio below 1 suggest a non-hollow (but neither perfectly compact) sphere structure, the ratio above 1 is usually assigned to particles with elongated shape. ${ }^{11,28}$

\section{Tunable resistive pulse sensing (TRPS)}

In TRPS, nanoparticles are directed though a nanopore as a result of electrophoresis. Fig. 3A shows the number-based distribution obtained by TRPS. The particle size distribution

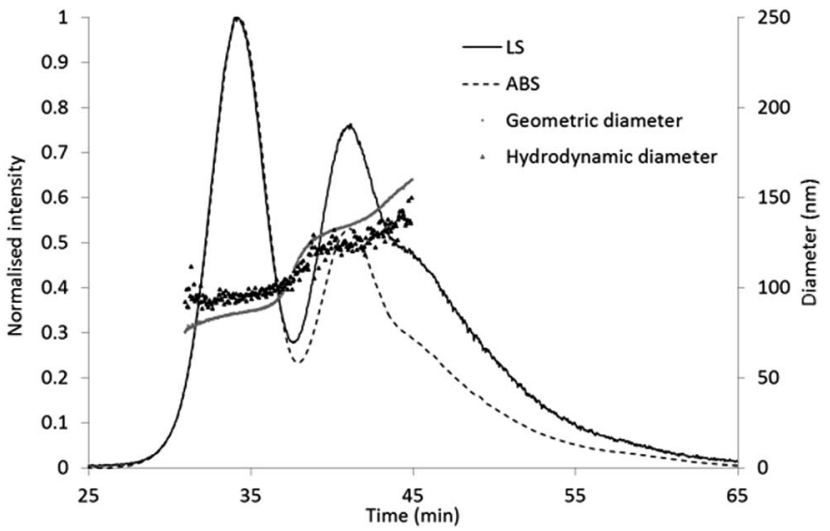

Fig. 2 Online FFF-UV-Vis-MALS-DLS results. Absorption signal: dashed line, light scattering: solid line, geometric diameter: grey diamonds, hydrodynamic diameter: dark, open triangles.

revealing one main population of particles with an average size of around $87 \mathrm{~nm}$ (mean = mode obtained by fitting the NP population with a normal distribution), possesses a Gaussian shape, with two additional secondary peaks centred at $106 \mathrm{~nm}$ and $115 \mathrm{~nm}$ (mean = mode obtained by fitting the NP populations with two normal distributions). The $x_{50}$ median derived from the number based cumulative distribution is $88 \mathrm{~nm}$ (see Table 2).
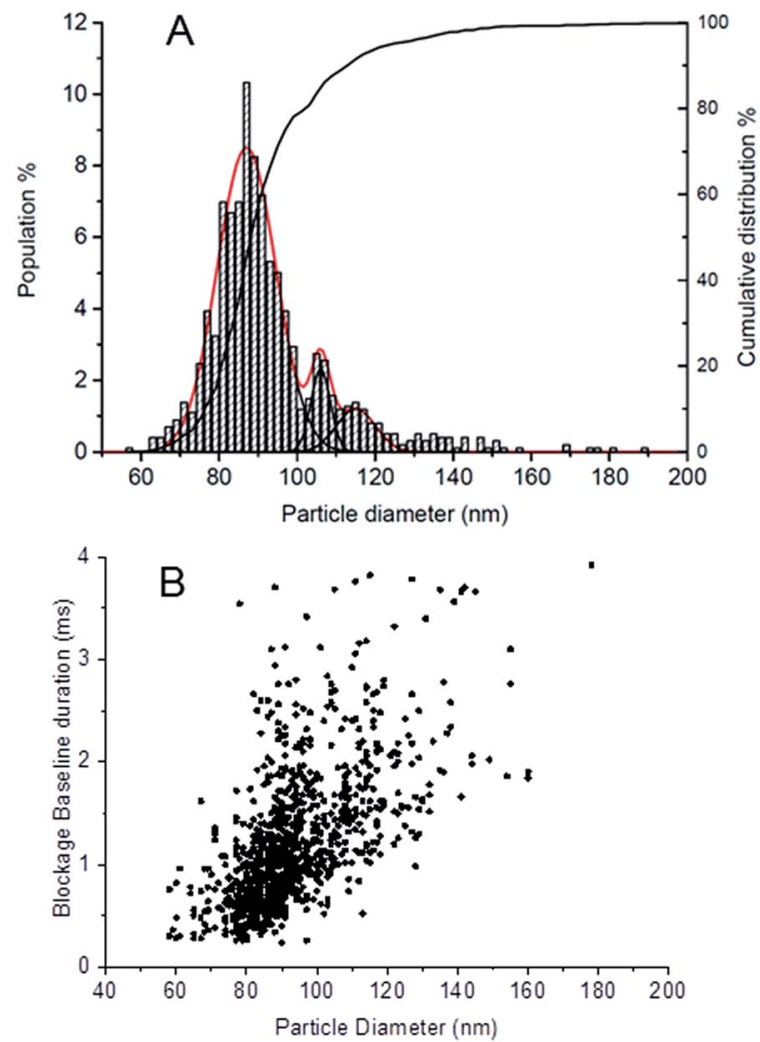

Fig. 3 TRPS results: (A) number based size distribution obtained from TRPS analysis. Solid lines: Gaussian distribution fits of the three main detected particle populations (red) and cumulative particle size distribution (black) (B) baseline blockage duration in function of the particle diameter. 
The smaller particle population detected by TRPS is presented in higher numbers, being the $78 \%$ of the total population. This result is consistent with the elution profile showed by FFF-MALS, confirming the accuracy of TRPS technique in resolving complex multimodal particle populations. ${ }^{7}$

From TRPS analysis it is possible to obtain some indirect information on the shape of the particles, as recently shown by Sikora and et al. ${ }^{29}$ TRPS measures the volume of the particles crossing the pore, which is then converted to the volume of an equivalent sphere. Therefore, the derived diameter for particle dimers and trimers will be the diameter associated to a sphere of equivalent volume, which is 1.26 and 1.44 times the average diameter (mode) of the pristine particles. The diameters of the larger populations measured in our study are 1.25 (106/85) and $1.35(115 / 85)$ times the measured diameter for the smaller particles. Those values are pretty close to the expected ones; suggesting that the second and the third peaks may be associated to nanoparticle dimers and trimers.

An additional indication of the presence of nanoparticle multimers can be derived from the analysis of the nanoparticle speed through the pore. Indeed, in TRPS the particle speed through the pore is driven by particle's shape (e.g. spherical vs. elongated), and surface charge. The software calculates the time each particle has travelled within the pore (blockage baseline duration), which is inversely proportional to particle speed, by measuring the resistive pulse signal generated by a transient reduction of the ionic current in the pore. ${ }^{30}$ Provided that the
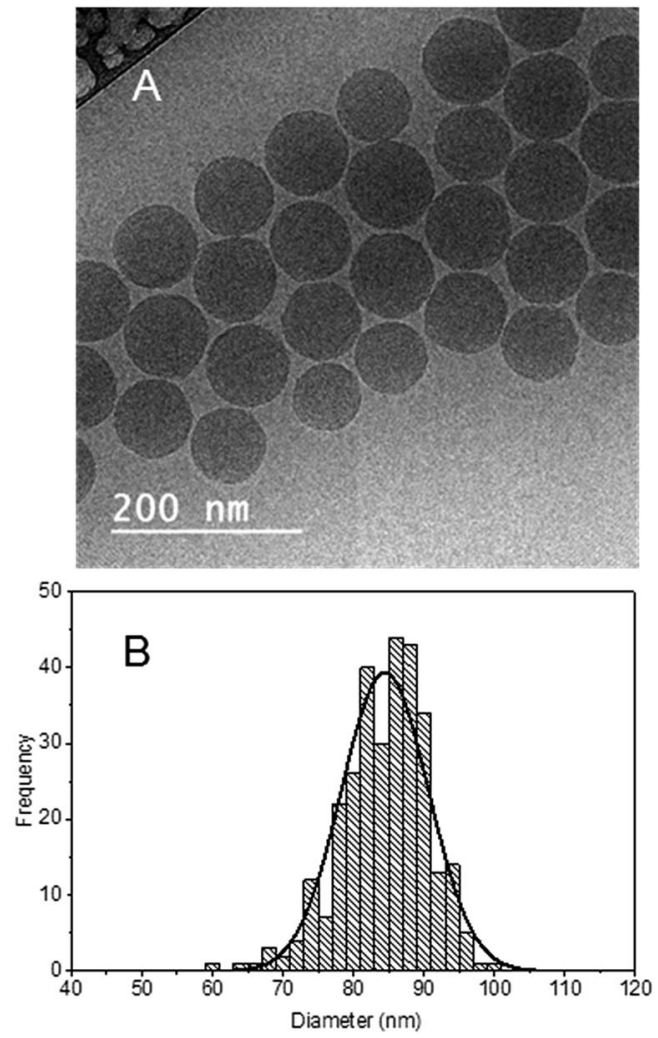

Fig. 4 (A) Transmission electron micrograph, scalebar represents $200 \mathrm{~nm}$. (B) Histogram of particle size distribution based on Cryo-TEM image analysis, solid line: Gaussian distribution fit. surface charge of the different populations does not vary significantly (which is a reasonable hypothesis in our case), we were expecting to measure the same speed for the three populations.

Surprisingly, we noticed a slight increase in the blockage time of the pore for the different populations (Fig. 3B), implying a reduction in particle speed with size. The fact that populations of 105 and $115 \mathrm{~nm}$ spend more time in the nanopore compared to the main population of $87 \mathrm{~nm}$, suggests that the larger particles may possess more elongated shapes.

The TRPS results support the assumption derived by the calculation of shape factors from FFF-MALS-DLS. However, since both FFF-MALS-DLS and TRPS can provide only indirect information on particle shape, direct imaging of particle morphology by electron microscopy is needed to confirm our hypothesis.

\section{TEM analysis}

Considering that NTA, TRPS and FFF separation results lead to the conclusion that there are two or three particle populations with different size, Cryo-TEM images might be surprizing at the first glance (Fig. 4A).

The micrographs show spherical particles with fairly similar diameter. The results of the image analysis indicates a rather monomodal size distribution with an average diameter of about $85 \mathrm{~nm}$ (Fig. 4B). Moreover, the diameter is below $100 \mathrm{~nm}$ for all
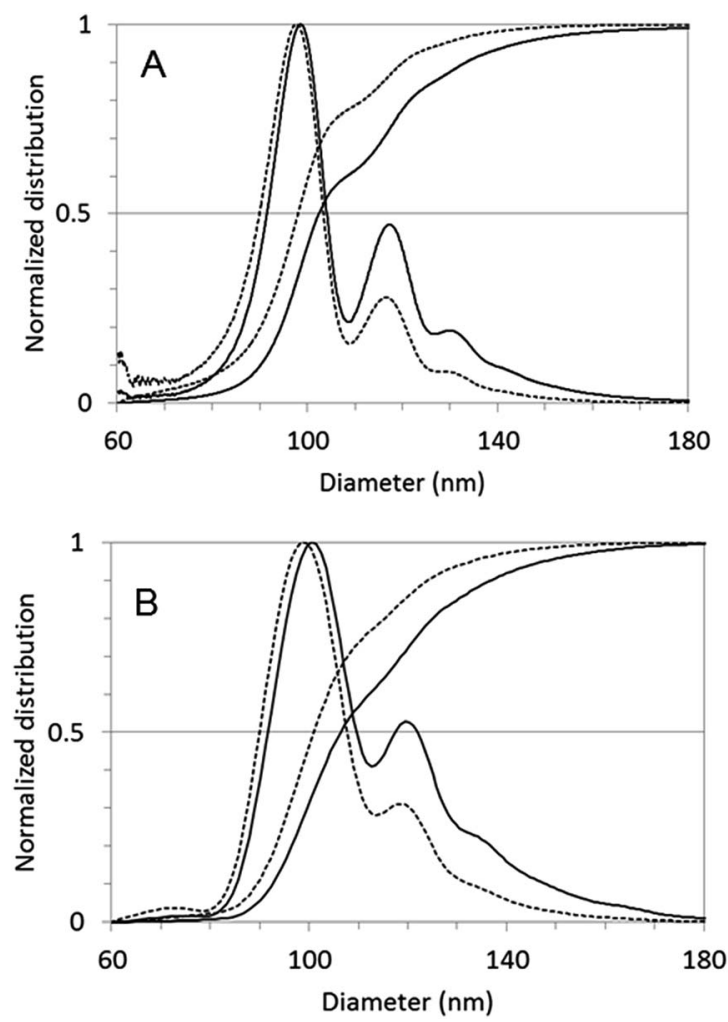

Fig. 5 Normalised mass (solid line) and number (dotted line) based particle size distribution measured by centrifugal methods. (A) CPS, (B) AUC results. 
Table 1 Mass based size distribution modes corresponding to the first two particle populations, calculated ratio of the apparent diameters $\left(D_{\text {app }}\right)$ and theoretical apparent diameter of rigid sphere dimers (theoretical $D_{\text {app, } 2 \text { ) }}$

\begin{tabular}{lllll}
\hline Method & $D_{\text {app }, 1}(\mathrm{~nm})$ & $D_{\mathrm{app}, 2}(\mathrm{~nm})$ & $D_{\mathrm{app}, 2} / D_{\mathrm{app}, 1}$ & $\begin{array}{l}\text { Theoretical } \\
D_{\mathrm{app}, 2}(\mathrm{~nm})\end{array}$ \\
\hline CPS & 98 & 118 & 1.2 & 117.5 \\
AUC & 100 & 120 & 1.2 & 119.9
\end{tabular}
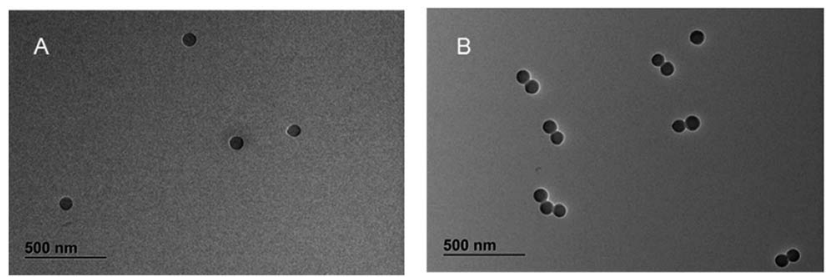

Fig. 6 (A) Single particles in the fraction of the first FFF peak and (B) particle aggregates in the second FFF peak.

observed particles, identifying the sample as nanomaterial (according to the EC recommendation on definition).

Confronting the TEM images with the different shape factors of the two particle populations obtained by FFF-MALS and with the difference in NPs speed obtained by TRPS, suggests that the second population corresponds to NPs that are not simply larger, but have a different, more elongated shape - they are most probably dimers or multiple aggregates.

\section{Centrifugal methods}

Centrifugal methods confirm our hypothesis regarding dimers. Both CPS and AUC analyses (Fig. 5) revealed a multimodal distribution with peak diameters associated to the first $\left(D_{\text {app }, 1}\right)$ and second peak $\left(D_{\text {app }, 2}\right)$ collected in Table 1 .

Dimers, due to their elongated shape, have to face more friction during sedimentation than perfect spheres and accordingly - sediment slower than a sphere of the same mass. Apparent Stokes diameter of a dimer $\left(D_{\mathrm{app}, 2}\right)$, corresponding to the diameter of an equivalent sphere sedimenting at the same speed, has a well-defined correlation to the apparent diameter of the constituting monomers $\left(D_{\mathrm{app}, 1}\right)$. The observed $D_{\mathrm{app}, 2} /$ $D_{\text {app }, 1}$ ratio in case of homodimers of rigid nanospheres seems to be quite independent from the material and in our case even from the centrifugal measurement method. The empirical correlation suggested by Bondoc and Fitzpatrick ${ }^{31}$ for small aggregates made of $N \leq 6$ adenovirus particles was shown to work very well also in case of other nanomaterials, including silica NPs ${ }^{1}$ and BSA coated polypyrrole particles. ${ }^{32}$

$$
D_{\text {app }, N}=\left(0.518+0.481 N^{1 / 2}\right) D_{\text {app }, 1}
$$

The $D_{\text {app }, 2} / D_{\text {app }, 1}=1.2$ ratio calculated from this equation for dimers is matching perfectly with the results of our centrifugal sedimentation experiments.

Gaussian fit of the differential mass distribution (CPS data) gives also good fit of theoretical diameters for the peaks at higher sizes (ESI, Fig. S7†). Trimers appear at slightly smaller diameter than expected, most probably because of the aggregates with unusual, close-to-linear morphology shown also in Fig. 6B.

\section{FFF-TEM combination}

Final confirmation of our hypothesis is provided by the TEM micrographs of the collected FFF fractions (Fig. 6) showing single particles in the fraction belonging to the first while dimers and trimers appearing in the second elution peak.

As (according to the information from the supplier) the PA02N particles did undergo a surface conversion $(-\mathrm{COOH}$ to $-\mathrm{NH}_{2}$ ) to obtain the amine function, most probably chemically induced cross linking occurred during the functionalisation, producing dimers and trimers.

\section{Dimer recognition by the various techniques}

Table 2 collects the diameter mode and median values of the number based size distributions obtained using the various nanoparticle characterisation techniques. The variability of size values reflects also the different nature of the determined diameters. As described above, TEM image analysis was done measuring Feret diameters, DLS and NTA (PTA) determine

Table 2 Diameter mode and median values of the number based size distributions obtained using the various nanoparticle characterisation techniques

\begin{tabular}{lllll}
\hline Method & Mode $1(\mathrm{~nm})$ & Mode 2 $(\mathrm{nm})$ & $\begin{array}{l}x_{50,0} \text { median } \\
(\mathrm{nm})\end{array}$ & Dimer recognition \\
\hline DLS & $79^{a}$ & n.a. & 80 & NO \\
PTA (NTA) & 95 & 133 & 135 & NO \\
TRPS & 87 & 105 & 88 & YES $^{b}$ \\
Cryo-TEM & 84 & n.a. & 84 & NO \\
FFF-MALS-DLS & n.a. & n.a. & n.a. & YES \\
FFF-TEM & n.a. & n.a. & n.a. & YES $^{b}$ \\
CPS & 98 & 117 & 101 & YES $^{b}$ \\
AUC & 99 & 119 & YES $^{b}$
\end{tabular}

${ }^{a}$ Mode of log scale differential size distribution. ${ }^{b}$ Indirect dimer recognition based on mathematical or empirical model. 
hydrodynamic diameters, MALS provides geometric diameters, TRPS calculates diameters from the apparent particle volume, and centrifugal techniques compute Stokes diameters from the sedimentation speed of equivalently behaving spheres. As a result, the apparent diameter of particle dimers is never simply the double of the diameter of monomers. Nevertheless, the presence of dimers can be forecasted by analysing the ratio of geometric and hydrodynamic radii (FFF-MALS-DLS) or by applying empirical models as in the case of TRPS and centrifugal methods. Finally, dimers were definitely identified by TEM after separation of particle populations by FFF.

\section{Conclusions}

As we demonstrated above in case of a representative nanomaterial example, no unique characterisation method can unquestionably reveal particle dimers. Even direct visualization imaging techniques as transmission electron microscopy and Cryo-TEM can fail to recognise them because of possible particle aggregation - if nanoparticles adopt preferred orientation on the grid. In our case, the polystyrene beads were concentrated near the border of the open areas of the mesh structure of the lacey carbon. The preferred orientation adopted by the particles on the borders of the carbon support of the grid $^{22}$ did not allow a reliable statistical analysis of single particles, dimers or larger aggregates by direct Cryo-TEM observations. However, combined with a separating and characterising systems, like FFF-UV-Vis-MALS-DLS, centrifugal methods, TEM analysis can definitely identify small particle aggregates. Indubitably, the introduction of online separation method prior to scattering detection techniques (FFF-UV-VisMALS-DLS measurement) provides more accurate size information about the particles than the batch mode DLS and the ratio of the geometric and hydrodynamic radius gives a good hint about particle shape.

PTA (NTA), TRPS and centrifugal methods were also capable to resolve the multimodal size distribution of the sample, but with different performance. PTA (NTA) might underestimate the amount of monomers simply because of the lower efficiency of tracking small objects in the presence of larger (shinier) ones, while TRPS, CPS and AUC had sufficient resolution to detect the three particle populations in comparable, and probably more realistic, proportions. TRPS analysis also gave multiple indirect indications of the presence of elongated particles - the multimers - associated to larger particles, e.g. by calculating the ratio between the diameters associated to multiple populations in the sample and also by observing a reduction of NP speed through the pore for larger particles.

Centrifugal methods, such as CPS and AUC that combine separation and detection steps in one measurement, provide very realistic size distribution results, even if the calculation of number based size distributions requires conversion steps from the originally recorded data.

Importantly, CPS and AUC not only separate particles with good size resolution at the nano definition borderline size, but also give indications of the presence of particle aggregates by the recognition of typical size distribution patterns. As the peaks of dimers and small $N$ element rigid multimers appear with typical $D_{\text {app }, N} / D_{\text {app, } 1}$ in the mass based size distribution independently from the composition of the material analysis of the size distribution patterns might be a simple and efficient tool to indicate aggregates. ${ }^{1,31,32}$

In special cases like this, the correct recognition of the nature of the multimodal distribution has a strong effect on the classification of the sample as nano or non-nano material. Considering that the second peak belongs to particle dimers, and the smaller diameter of these dimers is equal to the diameter of particle monomers, both centrifugal methods would classify the sample as nano material - in accordance with the TEM results.

\section{Acknowledgements}

This research was in part funded by the project EUNCL 2015, a European Union's Horizon 2020 Research and Innovation Programme under grant agreement No. 654190. We thank Dr Camille Roesch (Izon Sciences, Lyon) for the helpful discussions and advices on TRPS to Dr Isaac Ojea for practical help in TEM imaging and to Dr Agnieszka Mech for proofreading the article.

\section{References}

1 D. Zerrouki, B. Rotenberg, S. Abramson, J. Baudry, C. Goubault, F. Leal-Calderon, D. J. Pine and J. Bibette, Langmuir, 2006, 22, 57-62.

2 L. Li, T. Liu, C. Fu, L. Tan, X. Meng and H. Liu, Nanomedicine, 2015, 11, 1915-1924.

3 M. H. Jang, S. Lee and Y. S. Hwang, PLoS One, 2015, 10, e0143149.

4 R. Landsiedel, E. Fabian, L. Ma-Hock, B. van Ravenzwaay, W. Wohlleben, K. Wiench and F. Oesch, Arch. Toxicol., 2012, 86, 1021-1060.

5 European Commission, Journal, 2011/696/EU.

6 F. Varenne, A. Makky, M. Gaucher-Delmas, F. Violleau and C. Vauthier, Pharm. Res., 2016, 33, 1220-1234.

7 W. Anderson, D. Kozak, V. A. Coleman, A. K. Jamting and M. Trau, J. Colloid Interface Sci., 2013, 405, 322-330.

8 L. Calzolai, D. Gilliland, C. P. Garcia and F. Rossi, J. Chromatogr. A, 2011, 1218, 4234-4239.

9 S. Lee, S. P. Rao, M. H. Moon and J. C. Giddings, Anal. Chem., 1996, 68, 1545-1549.

10 S. Kosmella, J. Venus, J. Hahn, C. Prietzel and J. Koetz, Chem. Phys. Lett., 2014, 592, 114-119.

11 P. Iavicoli, P. Urban, A. Bella, M. G. Ryadnov, F. Rossi and L. Calzolai, J. Chromatogr. A, 2015, 1422, 260-269.

12 V. Filipe, A. Hawe and W. Jiskoot, Pharm. Res., 2010, 27, 796810.

13 S. J. Sowerby, M. F. Broom and G. B. Petersen, Sens. Actuators, B, 2007, 123, 325-330.

14 N. C. Bell, C. Minelli, J. Tompkins, M. M. Stevens and A. G. Shard, Langmuir, 2012, 28, 10860-10872.

15 A. K. Pal, I. Aalaei, S. Gadde, P. Gaines, D. Schmidt, P. Demokritou and D. Bello, ACS Nano, 2014, 8, 9003-9015. 
16 M. Sivakumaran and M. Platt, Nanomedicine, 2016, 11, 21972214.

17 D. Kozak, W. Anderson, R. Vogel, S. Chen, F. Antaw and M. Trau, ACS Nano, 2012, 6, 6990-6997.

18 ISO, Journal, 2007.

19 A. Aimable and P. Bowel, Process. Appl. Ceram., 2010, 4, 157166.

20 J. L. Cole, J. W. Lary, T. P. Moody and T. M. Laue, Methods Cell Biol., 2008, 84, 143-179.

21 P. H. Brown and P. Schuck, Biophys. J., 2006, 90, 4651-4661.

22 R. F. Thompson, M. Walker, C. A. Siebert, S. P. Muench and N. A. Ranson, Methods, 2016, 100, 3-15.

23 J. B. Simonsen, N. E. A. Reeler, A. Fossum, K. A. Lerstrup, B. W. Laursen and K. Nørgaard, Nano Res., 2016, 9, 30933098.

24 V. Sokolova, A. K. Ludwig, S. Hornung, O. Rotan, P. A. Horn, M. Epple and B. Giebel, Colloids Surf., B, 2011, 87, 146-150.
25 C. Cascio, O. Geiss, F. Franchini, I. Ojea-Jimenez, F. Rossi, D. Gilliland and L. Calzolai, J. Anal. At. Spectrom., 2015, 30, 1255-1265.

26 P. Schuck and P. Rossmanith, Biopolymers, 2000, 54, 328341.

27 V. Kestens and G. Roebben, The certification of equivalent diameters of a mixture of silica nanoparticles in aqueous solution: ERM-FD102, European Commission, Joint Research Centre, Institute for Reference Materials and Measurements, Geel, 2014.

28 J. Lohrke, A. Briel and K. Mader, Nanomedicine, 2008, 3, 437452.

29 A. Sikora, A. G. Shard and C. Minelli, Langmuir, 2016, 32, 2216-2224.

30 D. Kozak, W. Anderson, R. Vogel, S. Chen, F. Antaw and M. Trau, ACS Nano, 2012, 6, 6990-6997.

31 L. L. J. Bondoc and S. Fitzpatrick, J. Ind. Microbiol. Biotechnol., 1998, 20, 317-322.

32 K. M. Au and S. P. Armes, ACS Nano, 2012, 6, 8261-8279. 